\title{
Women Who Have Sex with Women's Experiences with Healthcare System in Low-income Countries: Qualitative Findings from Dar-es-Salaam, Tanzania
}

\author{
Happiness P. Saronga, Jackline V. Mbishi, Saidah M. Bakar, and Switbert R. Kamazima
}

\section{ABSTRACT}

\begin{abstract}
Introduction: Women who have sex with women (WSW) have a right to access health care. Many studies have reported lower access to health services by sexual minorities in many parts of the world. This study explored WSW's experiences in accessing health care in Tanzania with the intention of determining specific issues facing WSW when accessing health care services.
\end{abstract}

\begin{abstract}
Methods: This study was cross-sectional descriptive, and retrospective conduced in Dar-es-Salaam region, the largest commercial city in Tanzania. Study population included WSW aged 18 years and above who met inclusion criteria. Data was collected using focus group discussions (FGDs), in-depth interviews (IDIs), observation, and life stories. Data analysis applied thematic analysis.
\end{abstract}

Results: Most WSW receive rightful health services from public and private health providers. However, transgender WSW face stigma, discrimination, and disrespect from some public health facilities. Private health care providers offer trust, privacy and confidentiality to WSW, although at a higher cost of services compared to public health facilities.

Conclusion: Negative experiences with care may discourage WSW from seeking care or fully disclosing health concerns to providers limiting the extent of services offered.

Keywords: Health care utilization, Sexuality, Sexually transmitted infections, Tanzania, Women's health, Women who have sex with women.

\begin{abstract}
Published Online: July 18, 2021
\end{abstract}
ISSN: $2736-5476$

DOI: $10.24018 /$ ejclinicmed.2021.2.3.82

\section{Happiness P. Saronga}

Behavioral Sciences Department, School of Public Health and Social Sciences, Muhimbili University of Health and Allied Sciences, Tanzania.

(e-mail: sarongahappiness ${ }^{\circledR}$ yahoo.com) Jackline V. Mbishi

Epidemiology and Biostatistics Department, School of Public Health and Social Sciences, Muhimbili University of Health and Allied Sciences, Tanzania.

Saidah S. Bakar

Community Health Department, School of Public Health and Social Sciences, Muhimbili University of Health and Allied Sciences, Tanzania.

Switbert R. Kamazima

Behavioral Sciences Department, School of Public Health and Social Sciences, Muhimbili University of Health and Allied Sciences, Tanzania.

\section{INTRODUCTION}

Women who have sex with women (WSW) engage in risky behaviors and practices that make them vulnerable for acquiring diseases, including sexually transmitted infections (STIs) [1]. WSW engage in promiscuity, bisexuality, unsafe sex, transactional sex, abuse of drugs and/or have partners who abuse drugs [1]-[6]. WSW are a 'bridge population' for transmission of STIs from the highest-risk groups (MSM, bisexual, IDUs, and FSWs) to the general population. Not only that, WSW have risk of non-communicable diseases (NCDs), for example, mental illness, cancer, diabetes, and heart problems, just like everyone else in the general population.

For these reasons WSW need health care to prevent and treat health problems just like people in the general population, if not more given their sexual risk profile [7]. Health and health care access are fundamental human rights for everyone. Many studies have reported lower access to health services by sexual minorities [7]-[10]. Barriers to access include stigma, discrimination, and lack of money, among others [7], [10], [11]. For example, findings from a research study on barriers to health care access among MSM in Tanzania reported lack of money as the primary factor for non-use of health care [9].

This study explored WSW's experiences in accessing health care in Tanzania with the intention of determining specific issues facing WSW when accessing health care services. There is no such information on WSW in Tanzania; results from this study may inform public health interventions and programs targeting WSW in Tanzania.

\section{MATERIALS AND METHODS}

This was a cross-sectional descriptive and retrospective formative study. This study was conducted in Ilala, Kinondoni and Temeke districts in Dar-es-Salaam region. Dar-es-Salaam is the largest commercial city in Tanzania, known to harbor persons from different backgrounds and lifestyles including those engaging in varied sexual behaviors and practices therefore allowed easier access to study participants. Inclusion criteria for the participants were age 
18 years and above, residence in Dar-es-Salaam for six (6) months or more; engagement in same-sex sex in the past year and/or in same-sex relationship/s; and knowledge of WSW's lived experiences and willingness to participate in the study.

Data collection was performed through focus group discussions (FGDs), in-depth interviews (IDIs), key informant interviews (KIIs) and life stories. Training on the study objectives and procedures, together with research ethics with regards to the key population to the research assistants (RAs) was conducted prior to data collection. Research permission and consent was sought from authorities and participants respectively. FGDs, KIIs and IDIs were audiorecorded, and research assistants took field notes as well. All tools were administered in Kiswahili; the national language spoken by almost everyone in Tanzania.

Transcription and translation of data was conducted. Data analysis applied thematic analysis where open coding approach was used using participants' language and combining emerging emic concepts with preconceived theoretical constructs. Ethical clearance for this study was granted by the Muhimbili University of Health and Allied Sciences (MUHAS) Institutional Review Board (IRB). Participation in the study was voluntary; participants gave a verbal consent for participation.

\section{RESULTS}

The objective of this study was to explore experiences of WSW in accessing health services. To get this information, we posed a question: What has been your experience with the healthcare system/services as a WSW? Majority of our study participants reported that WSW who are tomboys and transgender men and women have unfavorable experiences with the healthcare system in the country compared to the non-transgender WSW.

A participant aged 32, never married, a mother of one child and a sex worker, stated, "Whenever I feel unwell, I visit the public health facilities [because services are free] ... I have never had any challenge accessing [health] services, because I always present [dressing and body language] a female ... No one [a doctor or a nurse] can recognize I engage in same sex behaviors ... However, I hear the tomboys face some challenge due to their presentation (IDI, $H, 32$ years, 2021). Another participant aged 27, divorced, has O-level education and has sex with men, reported, "I usually present a female [dressing] whenever I visit health facilities ... I go to public facilities where services are free ... The healthcare providers treat me like any other client ... No one can tell I engage in same sex ... However, the tomboys tell us they are often stigmatized and discriminated ... Sometimes they are denied services ... It is a pity ... Our government does not respect our rights" (IDI, A, 27 years, 2021).

A participant, aged 35, divorced, a primary education graduate and engages in sex work, reported,

"The tomboys and transgender men and women face stigma and discrimination when they visit public health facilities seeking healthcare services ... Stigmatization follows from healthcare providers' recognition that their client presenting a male [dressing, low voice and haircuts] is actually a female ... At some facilities, healthcare providers assemble to witness what their colleague has encountered ...
Whenever this happens, the tomboys and transgender men and women just walk away without treatment, forcing them to undertake self-medication ... If they have money, they seek care services at private facilities, where no one cares provided you are able to cover the costs (IDI, B, 35 years, 2021).

Another participant aged 46, never married, a mother of two children and has sex with men, stated,

Please note, whenever I go to the hospital, I do not present a woman engaging in this business [female same sex] ... I present as any woman ... A straight woman in all aspects [dressing and body language] ... I normally visit the public facilities, Mwananyamala, Temeke or Muhimbili hospitals ... I visit these facilities for any health condition I have, like malaria, cough or headaches ... I have never been stigmatized or discriminated ... They do not know my sexual behaviors and practices... They regard me as any woman ... However, I am told the tomboys and transgender men and women face challenges because of their presentation" (IDI, G, 46 years, 2021).

The other participant aged 26, never married, a transgender man and a university graduate, had this story, "What I have experienced is that the bottom [women playing a female role in same sex relationship] access healthcare services at any facility in this country ... Including the public facilities, because they present as any heterosexual women ... It is us [transgender men and women and tomboys] who are stigmatized or discriminated at public facilities... Sometimes we are denied access to care we need leading some not to seek treatment or depend on self-medication, which is not good for their health ... However, we have found a way around this situation ... As one decides to go through transition [from a male to a female and vice versa], we identify our private doctors [mainly at private facilities] whom we trust and can share any concern regarding our missions ... They [doctors] assure us adhering to confidentiality ... They support us throughout this process [transition] (IDI, F, 26 years, 2021).

Another participant aged 28, never married, has O-level education and engages in sex work, reported, "We [the bottoms] seek healthcare from any public facility in the city ... We face no challenge ... They [healthcare providers] treat us like any other citizen ... We advise each other to go to the hospital for any ill health we feel ... However, it is the tomboys or transgender men and women [who present as men] who face stigmatization and discrimination at public health facilities" (IDI, E, 28 years, 2021). Presenting an example of the challenges the tomboys/transgender men and women face, the same participant had this to tell, "It happens that the tomboy has a problem that requires him to undress for the doctor's examination, say on the chest ... Usually, the care providers get astonished seeing they have breasts ... They [doctors] yell at them 'why do you present a male while you are a female ... You have breasts, you are a female' ... Sometimes the doctor may call his/her colleagues to witness what he/she has encountered ... Such behaviors force tomboys and transgender men and women to shun public facilities ... They either go to the private facilities or depend on self-medication" (IDI, E, 28 years, 2021).

A participant in the FGD, reported, "I shall present general experiences we have ... Say one [WSW] goes to [public] 
health facility suffering from mouth fungus ... The doctor will quickly ask, 'what did you do to get fungus in your mouth?' ... Depending how serious you feel, you have to tell the doctor the truth [I am a WSW] to get services... You tell him or her I practice oral sex with fellow women ... The doctor starts lecturing you on the side effects of oral sex and same sex ... We find this unnecessary and offending ... We avoid going back to these facilities ... We go to the private hospitals or depend on self-medication" (FGD_1, 2021).

We further asked our study participants to recommend on what should be done to improve WSW's access to healthcare services in Dar-es-Salaam region and the country at large. Participants' perspectives are as follows. A participant aged 35 , divorced, has primary level education and engages in sex work, stated, "I plead the government to recognize us and respect our rights ... We are human beings like those called the straight ... We know, we engage in risky sexual behaviors ... We need to be informed of the risks involved as we [the researchers and her] have discussed ... We need lubricants and other devices [dental dams and condoms] to protect ourselves from infections [HIV and STIS] ... As the government recognizes the existence of men having sex with men, should also recognize we [WSW] exist" (IDI, B, 35 years, 2021).

Another participant aged 26, never married, a transgender man and a university graduate, beseeched, "In my view, the healthcare providers should not stigmatize and discriminate us ... We are human beings as they are ... They have to keep any information we present confidential ... We pay government taxes ... They should treat us irrespective of our sexual orientation ... "Wasitunyanyapae" [healthcare providers should not stigmatize us] ... The fact is that no human being is 100 percent perfect ... They [healthcare providers] may be perceived abnormal in other contexts (IDI, F, 26 years, 2021).

Another participant aged 27, divorced, has O-level education and has sex with men, implored, "The healthcare providers should treat us like any other citizen ... The tomboys and transgender women are human beings too with the right to access healthcare at any health facility they visit ... The tomboys and transgender men and women should be respected and cared for like other patients" (IDI, A, 27 years, 2021). Another study participant aged 28, never married, has O-level education, and engages in sex work, pled, "I plead the healthcare providers to treat tomboys, transgender men and women with respect and provide them with equitable healthcare services just like any other patients" (IDI, E, 28 years, 2021).

\section{DISCUSSION}

WSW, who are not transgender, have access to rightful health services without any problems. Incidents of condemnation, stigma and discrimination from health care providers are experienced almost solely by transgender WSW (transgender men and women), who are sometimes denied services by care providers because of their obvious sexual behavior. These discriminative incidents toward the WSW have also been reported in past research in Tanzania [8]. The WSW behavior is frowned upon by some health care providers, which many cause negative experiences during health care utilization by WSW [8], [10]. Negative experiences may eventually precipitate sub-optimal utilization of services, non-utilization of services, and sometimes self-medication. Sub-optimal utilization may be in form of non-disclosure of sexual behavior. A study in Tanzania indicated stigma, discrimination, shame, mistreatment, and lack of confidentiality as factors for nondisclosure of sexual orientation to health providers by MSM during care, a decision that might have interfered with proper treatment [9]. Similar studies in East Africa and across the world have reported similar barriers to health care access among key population [10], [11].

Non-utilization of health care among WSW has negative implications to the Tanzania health system as WSW are a high-risk group for sexual infections, as well as other communicable diseases and NCDs [7]. For example, untreated HPV may lead to cervical and anal cancer, and untreated hepatitis may lead to liver cancer. Furthermore, self-medication may have short-term and long-term adverse side effects to an individual including adverse drug reaction, deadly drug interaction, and drug resistance. Consequently, WSW need equal access to SRH and non-SRH preventive and treatment services in order to promote positive health outcomes in the community. The WSW access to free services at public health facilities is a good thing for promoting health care access and positive health outcomes, however the reported stigma, discrimination, and disrespect among transgender in this study occurred exclusively in public health facilities. These negative experiences have bred WSW mistrust on the health care system and non-use of health care. Similar experiences of stigma, discrimination, and disrespect among key population have been reported in related research in Tanzania [8] and in Kenya [10].

Private health facilities seem to offer friendlier services to WSW (transgender) than public health facilities. WSW have more trust in private health care providers, and confidentiality is guaranteed, and this has also been reported in similar research on key population in Tanzania [8]. A study on access to sexual and reproductive health (SRH) among adolescent key population in Kenya showed that adolescents prefer private health care compared to public health care because of increased confidentiality, limited stigma and discrimination, and friendly services, among others [10]. Nonetheless, private providers are costlier than public health facilities; hence, not many WSW can afford, especially those not covered by health insurance. Financial barrier was reported as factor for non-access to health care among MSM in Tanzania in a similar study [8]. Although we did not explore health insurance status of study participants, many of the interviewed WSW are of low socio-economic status and may largely rely on free health care from public providers, making non-discriminatory access to health care extremely essential for this group.

The WSW call for recognition and equity in access to health care. They accept the riskiness of their behavior and vulnerability of contracting STIs; hence, call on the public health care providers to avoid prejudice and honor their duty of treating all people with dignity and fairness regardless of their sexual orientation. National treatment guidelines are in place for all populations; these guidelines feature key population specific guidelines [12]. Although the guidelines 
do not explicitly mention WSW, transgender men and women are explicitly covered in the guidelines (especially for HIV services). This means that WSW have the right to receive needed services as spelled out in the guidelines, the problem is with providers' negative perceptions towards WSW creating non-friendly environment that repel utilization of health services by transgender WSW. Non-utilization of services by WSW may slow down efforts of the health system in improving health care access and health outcomes for everyone.

\section{CONCLUSION}

Most WSW receive rightful health services from public and private health providers. However, transgender WSW face stigma, discrimination, and disrespect from some public health facilities. This discourages trust and health care access, promoting self-medication that can be detrimental to WSW's health. Private health care providers offer trust, privacy, and confidentiality to WSW, although at a higher cost of services compared to public health facilities. Negative experiences with care may discourage WSW from seeking care or fully disclosing health concerns to providers limiting the extent of services offered to WSW and eventually delaying efforts of achieving universal health care access and improving health outcomes for all in Tanzania.

\section{ACKNOWLEDGEMENTS}

We would like to express our sincere gratitude to the participants who willingly shared their experiences and research assistants for assisting in data collection. Also, we thank SIDA small grants scheme at MUHAS for providing funds that made this study possible.

\section{REFERENCES}

[1] Koh AS, Gómez CA, Shade S, Rowley E: Sexual risk factors among self-identified lesbians, bisexual women, and heterosexual women accessing primary care settings. Sex Transm Dis 2005, 32:563-569.

[2] Fethers K, Marks C, Mindel C, Estcourt CS: Sexually transmitted infections and risk behaviours in women who have sex with women. Sex Transm Inf 76345-349, 76:345-349.

[3] Marazzo JM, Gorgos LM: Emerging sexual health issues among women who have sex with women. Curr Infect Dis Rep 2012, 14:204211.

[4] Bailey J V., Farquhar C, Owen C, Mangtani P: Sexually transmitted infections in women who have sex with women. Sex Transm Infect 2004, 80:244-246.

[5] Gorgos LM, Marrazzo JM: Sexually transmitted infections among women who have sex with women. Clin Infect Dis 2011, 53(SUPPL. 3).

[6] Hughes C, Evans A: Health needs of women who have sex with women. Bmj 2004, 328:464.

[7] Delany-Moretlwe S, Cowan FM, Busza J, Bolton-Moore C, Kelley K, Fairlie L: Providing comprehensive health services for young key populations: Needs, barriers and gaps. J Int AIDS Soc 2015, 18:29-40.

[8] Human Rights Watch: "Treat Us Like Human Beings” Discrimination against Sex Workers, Sexual and Gender Minorities, and People Who Use Drugs in Tanzania. 2013.

[9] Magesa DJ, Leshabari M: Perceived barriers to access available health services among men who have sex with men in Dar es Salaam, Tanzania. Tanzan J Health Res 2017, 19:1-8.

[10] Robert K, Maryline M, Jordan K, Lina D, Helgar M, Annrita I, Wanjiru M, Lilian O: Factors influencing access of HIV and sexual and reproductive health services among adolescent key populations in Kenya. Int J Public Health 2020, 65:425-432.

[11] Ssekamatte T, Isunju JB, Naume M, Buregyeya E, Mugambe RK, Wanyenze RK, Bukenya JN: Barriers to access and utilisation of HIV/STIs prevention and care services among trans-women sex workers in the greater Kampala metropolitan area, Uganda. BMC Infect Dis 2020, 20:1-15.

[12] NACP: Comprehensive Package of HIV Interventions for Key Population. 2014(September). 